Portland State University

PDXScholar

Physics Faculty Publications and Presentations

Physics

8-2019

\title{
The Fourier Spectrum of a Singing Wine Glass
}

\author{
Reuben Leatherman \\ Portland State University \\ Justin Charles Dunlap \\ Portland State University, jcdunlap@gmail.com \\ Ralf Widenhorn \\ Portland State University, ralfwidenhorn@pdx.edu
}

Follow this and additional works at: https://pdxscholar.library.pdx.edu/phy_fac

Part of the Physics Commons

Let us know how access to this document benefits you.

\section{Citation Details}

Leatherman, R., Dunlap, J. C., \& Widenhorn, R. (2019). The Fourier spectrum of a singing wine glass.

American Journal of Physics, 87(10), 829-835.

This Article is brought to you for free and open access. It has been accepted for inclusion in Physics Faculty Publications and Presentations by an authorized administrator of PDXScholar. Please contact us if we can make this document more accessible: pdxscholar@pdx.edu. 


\section{The Fourier spectrum of a singing wine glass}

Reuben Leatherman, Justin C. Dunlap, and Ralf Widenhorn

Citation: American Journal of Physics 87, 829 (2019); doi: 10.1119/1.5124230

View online: https://doi.org/10.1119/1.5124230

View Table of Contents: https://aapt.scitation.org/toc/ajp/87/10

Published by the American Association of Physics Teachers

\section{ARTICLES YOU MAY BE INTERESTED IN}

Dangerous limits

American Journal of Physics 87, 775 (2019); https://doi.org/10.1119/1.5125214

Thermodynamics of Benford's first digit law

American Journal of Physics 87, 787 (2019); https://doi.org/10.1119/1.5116005

Single-pixel camera

American Journal of Physics 87, 846 (2019); https://doi.org/10.1119/1.5122745

The forced harmonic oscillator: Coherent states and the RWA

American Journal of Physics 87, 815 (2019); https://doi.org/10.1119/1.5115395

Laminar viscous flow through pipes, related to cross-sectional area and perimeter length

American Journal of Physics 87, 791 (2019); https://doi.org/10.1119/1.5113573

Uncle Jesse and the seven "early career" ladies of the night ${ }^{1}$

American Journal of Physics 87, 778 (2019); https://doi.org/10.1119/1.5122880

\section{AAPT Learn about the newest AAPT member benefit}




\title{
The Fourier spectrum of a singing wine glass
}

\author{
Reuben Leatherman, Justin C. Dunlap, and Ralf Widenhorn \\ Department of Physics, Portland State University, P.O. Box 751, Portland, Oregon 97207
}

(Received 21 May 2019; accepted 9 August 2019)

\begin{abstract}
The phenomenon of the singing wineglass is familiar to many. Most people have run a finger along the rim of a wine glass with the right speed and pressure to get it to whistle a tone or perhaps heard a glass harmonica being played. However, have you ever noticed and wondered why the vibrations caused by a finger on a glass produce a pulsating sound, rather than a steady, constant-amplitude vibration? Further exploration reveals that the sound and pulsations of a wine glass vary depending on the way the wine glass is stimulated. In this paper, we investigate and model the characteristic sounds produced by three different cases: the pulsating sound exhibited by a finger run along the rim of a wine glass, the steady tone produced by a stationary finger on a rotating wine glass, and the decaying pulsations exhibited by a struck rotating wine glass. Analyzing the qualitative differences among these three cases provides opportunities for students to hone experimental, modeling, and data analysis skills in an intermediate level undergraduate experimental physics course. (c) 2019 American Association of Physics Teachers.

https://doi.org/10.1119/1.5124230
\end{abstract}

\section{INTRODUCTION}

The oscillations which occur through the distortion of the rim of a vibrating wine glass have been well studied. ${ }^{1}$ The physics of wine glass acoustics was first described by the Nobel Laureate Lord Rayleigh in $1894 .^{2}$ In his analysis of the deformation of thin elastic shells, he found that the frequency of vibration of a struck glass was the same as the frequency produced by a finger run along the glass rim. ${ }^{2}$ The vibration produced by a rotating finger is caused by friction between the finger and glass rim producing tangential vibrations in the glass most extreme at antinodes and correspondingly into radial motion at nodes. ${ }^{2,3}$

The case of a finger on a rotating glass is further investigated by Spurr, who describes how ringing is induced by a rubber finger on a glass rim. ${ }^{3}$ According to his analysis, ringing only occurs when a liquid lubricant is used, causing dynamic friction between the rim of the glass and the rubber finger to become less than the static friction. Ringing only occurs when a lubricant is used along with a sufficiently high rotational velocity of the finger, resulting in stick-slip behavior.

The principal modes of vibration occur via propagation of bending waves around the glass rim, which define $2(n+1)$ stationary nodes and antinodes around the circumference, with $n$ being an integer number. The lowest harmonic is $n$ $=1$, where the rim of the glass changes from circular to elliptical twice per cycle. This harmonic typically produces the dominant signal and is heard best. Fenkner found that the modal frequency of this vibration is roughly proportional to the glass thickness and inversely proportional to the square of the glass radius and is roughly independent of the height of the glass bell. ${ }^{4}$ These modes, also known as bell modes, are similar to the vibrational modes of a flat plate and can be analyzed using a modified version of Chladni's law. ${ }^{5}$ Rather than the modal diameters of a flat plate, however, nodes take the form of meridians running vertically along the height of the glass bell. In higher vibrational modes, nodal circles running along the glass bell's circumference are also present. ${ }^{6}$

Through computational modeling and experiment, we explore the phenomenon in which the vibrational nodal meridians are not stationary relative to their initial position along the rim of the wine glass. As a finger moves around the rim of the glass, the region of maximum vibration follows the finger, resulting in a sound that pulsates in a manner similar to the beats generated by two different frequency sound sources. $^{7}$ In the case of the rotating finger, the pulsation rate depends upon the angular speed of the finger rather than the frequency difference in the beat phenomena.

Upon deeper analysis, these phenomena are not surprising, but are non-obvious and provide ample opportunities for teaching students about standing waves, sound, interference, and Fourier Transforms in undergraduate labs for physics majors or engineering students. We believe that this paper highlights an area of acoustics that can be both instructive and thought provoking in the context of experimental physics education.

\section{EXPERIMENTAL SETUP}

To study this phenomenon quantitatively, wine glass spinners were designed that allowed us to control the frequency of rotation in two different configurations.

\section{A. Rotating finger}

The first setup rotated the finger, while the wine glass (we used a wine glass with a $4.0 \mathrm{~cm}$ radius at the rim of the glass) remains stationary. As a substitute for a real finger, we mounted a piece of stiff rubber tubing to the turntable on a vertical metal rod (see Fig. 1). We manually adjusted the speed of rotation of the rubber finger and its pressure on the glass rim in order to achieve a steady tone.

\section{B. Rotating glass}

The second piece of apparatus used a wine glass mounted to a turntable (see Fig. 2). The angular speed of the turntable was controlled with a variable power supply, while a stationary finger was pressed to the rim of the spinning glass to produce a tone. Additionally, instead of a stationary finger held to the rotating glass, a pencil was used to tap the glass as it rotated, which created a tone that decayed after a few seconds. Two microphones positioned radially in the plane of the glass rim recorded the tone. 


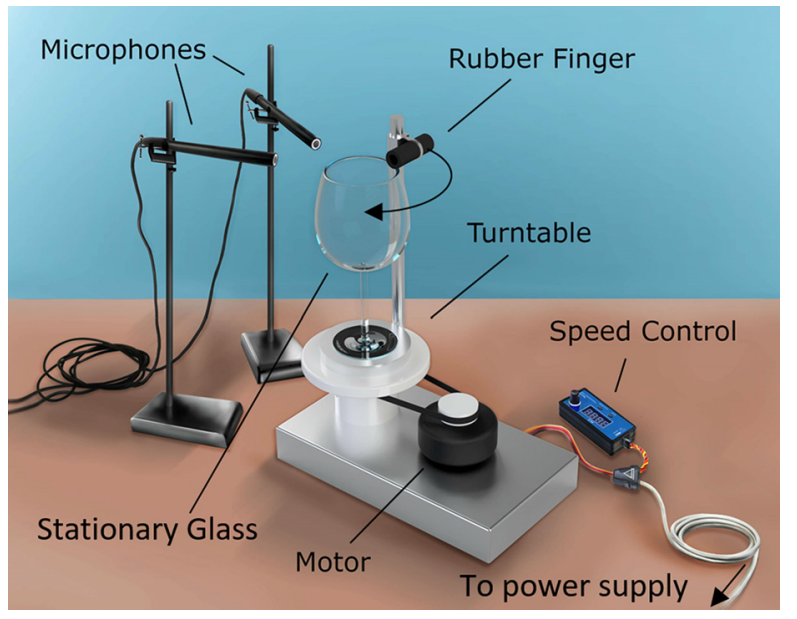

Fig. 1. Vibrations are produced by a rubber finger rotated around a stationary glass rim at a variable speed.

For both setups, we used Vernier microphones and rotary motion sensors with LoggerPro to collect the sound and rotational speed data. ${ }^{8}$ The low noise Turnigy Power Systems gimbal motor 5208 was connected to a speed control unit and a DC power supply.

\section{EXPERIMENTAL DATA}

\section{A. Rotating real finger}

The first experiment uses a real finger rotating around the rim of a stationary wine glass to produce a tone with a fundamental frequency of $488.8 \mathrm{~Hz}$ that is picked up by two radially positioned microphones coplanar with the glass rim and azimuthally offset by $45^{\circ}$ from each other. In our case, the recorded signals between the two microphones were found to have a $\pi$ phase offset, independent of frequency. This offset was time invariant allowing us to investigate the differences between in-phase and out-of-phase behavior between the waveforms of the two signals. (When conducting this part of the experiment, students need to make sure that they investigate the relative phase of their two microphones, for example, by placing them next to each other far from a

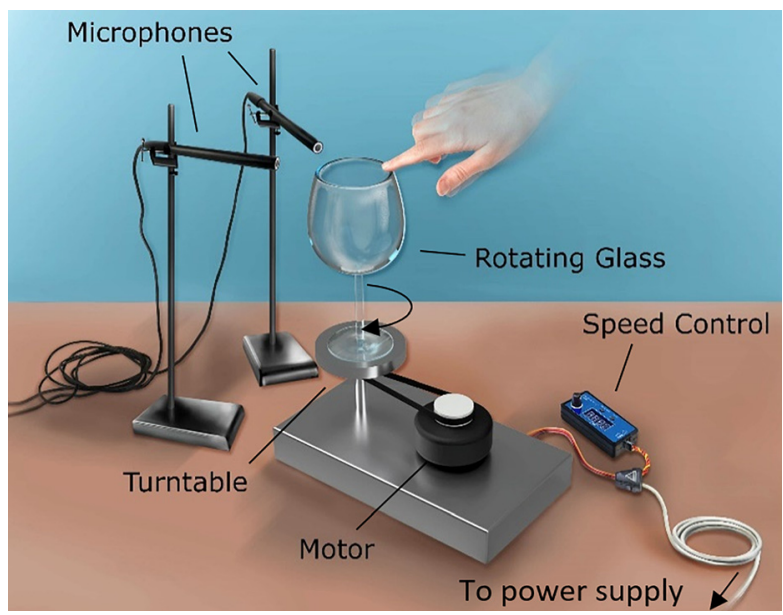

Fig. 2. Vibrations are produced by the pressure of an actual finger on the rim of a glass spun with a variable-speed turntable while the finger is stationary. Alternatively, decaying vibrations are produced when the spinning glass is tapped with a pencil.

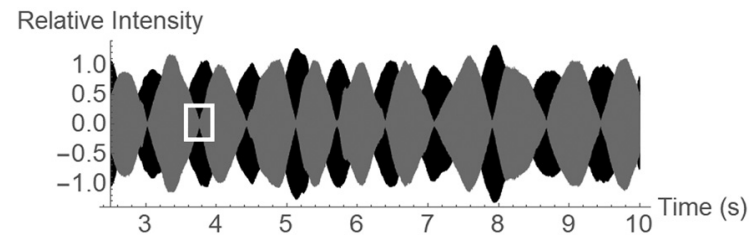

Fig. 3. Sound signals from a wet finger rotating around the rim of a wine glass. The signals are measured by two microphones offset by $45^{\circ}$. The boxed region is examined in more detail in Fig. 4.

known signal frequency source.) The resulting beat-like phenomenon is illustrated in Fig. 3, which depicts the envelope of the amplitude modulated signals picked up by the two microphones. Average amplitudes recorded by each microphone are normalized to the same level for all following data except Figs. 9 and 10. Figure 3 clearly depicts exactly out of phase sound patterns between the two microphones.

A difference in phase can also be observed between the two waveforms, depending on which part of the sound envelope is chosen. The phase shift switches between 0 and $\pi$ each time either of the waves' amplitudes pass a minimum. Figure 4 illustrates one of these cases in a small time interval of the signals shown in Fig. 3.

In Fig. 4, the two waves are completely out of phase before $3.76 \mathrm{~s}$ and become in phase shortly after the minimum past $3.76 \mathrm{~s}$. This minimum, therefore, is the dividing line between the two phase shifts. Similar transitions can be observed each time a minimum of either signal is passed.

\section{B. Rotating rubber finger}

In the second experiment, we observe the sound picked up by two microphones when the rubber finger slides around the rim of the glass at a constant speed, as shown in Fig. 1. This experiment serves to investigate the relationship between the pulsating sound and the angular speed of the stimulating rotating finger.

When the microphones are positioned $45^{\circ}$ apart, the signals picked up by the two receivers have sound amplitude patterns that are completely out of phase (see Fig. 5). A secondary amplitude pattern with a period of four times the main amplitude pattern is also visible.

Next, the microphones are repositioned to form a $90^{\circ}$ angle, with both pointing towards the center of the glass. When the microphones are positioned $90^{\circ}$ apart, the signals show pulsation sound patterns that are in phase. Figures 5 and 6 reveal a relationship between the microphones' separation angle and the phase shift of the observed amplitude oscillation.

The Fourier spectrum of the experimental finger rotation data shown in Fig. 7 reveals two major frequency peaks at $486.9 \mathrm{~Hz}$ and $490.2 \mathrm{~Hz}$. Two pairs of smaller side peaks accompany each of the larger peaks symmetrically on either side at $486.0 \mathrm{~Hz}, 487.8 \mathrm{~Hz}, 489.4 \mathrm{~Hz}$, and $491.0 \mathrm{~Hz}$. These

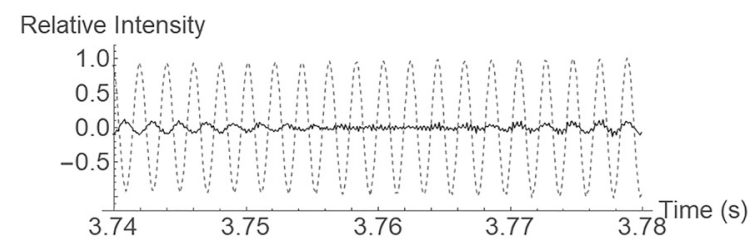

Fig. 4. Waveforms from two microphones offset by $45^{\circ}$ centered on the amplitude zero of the solid-line signal occurring at approximately $3.76 \mathrm{~s}$. 


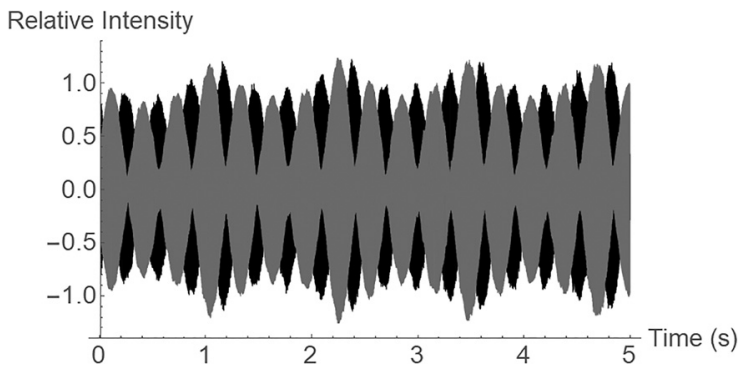

Fig. $5.45^{\circ}$ microphone offset-finger rotating at $5.18 \mathrm{rad} / \mathrm{s}$.

symmetric side peaks occur consistently across all rotatingfinger data runs with a pronounced 4-pulse secondary oscillation (as in Fig. 5). Besides these peaks, miscellaneous small signals and noise are created by other minor resonances of the glass and do not appear consistently in all of our rotating-finger data. Therefore, we conclude that they are not significant to the phenomena of interest. Importantly, we observe that the difference between the two major frequency peaks $-3.3 \mathrm{~Hz}$ or $20.7 \mathrm{rad} / \mathrm{s}$ - is four times the rotational frequency of the glass, which in this case was $5.18 \mathrm{rad} / \mathrm{s}$. This factor of four was observed in all rotating-finger data runs, at a variety of rotational speeds.

\section{Rotating glass with a stationary finger}

When the glass rotates instead of the finger, we observe something different. In this case, a real finger can be used, or the rubber finger can be held stationary relative to the glass rim.

Unlike the case with the rotating finger, Fig. 8 shows a sound intensity that is not characteristic of a beat-like signal. The periodic fluctuations in both amplitudes correspond to the slight variations in pressure by the imperfectly flat rim of the glass as it rotates on the stationary finger and scales with the rotational speed of the glass. In contrast to the paired frequencies of similar magnitude observed with a rotating finger, the fundamental resonance of $488.8 \mathrm{~Hz}$ remains the predominant frequency in the Fourier spectrum for this case. The Fourier spectrum shown in Fig. 9 also depicts several pairs of symmetrc side peaks at a variety of magnitudes. The closest pairs are spaced roughly $1.56 \mathrm{~Hz}$ (or $9.80 \mathrm{rad} / \mathrm{s}$ ) away from the fundamental frequency peak, corresponding to the rotational speed of the glass causing periodic pressure and amplitude variation. Other peak pairs are spaced at integer multiples of the rotational frequency away from the main peak, indicating an unevenness of the glass rim that oscillates more than once along its circumference. Various small peaks likely correspond to low frequency amplitude variation caused by inconsistancy

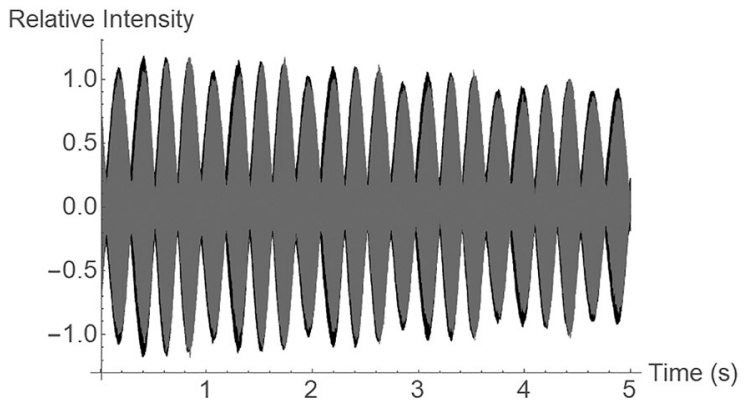

Fig. 6. $90^{\circ}$ microphone offset-finger rotating at $6.97 \mathrm{rad} / \mathrm{s}$.

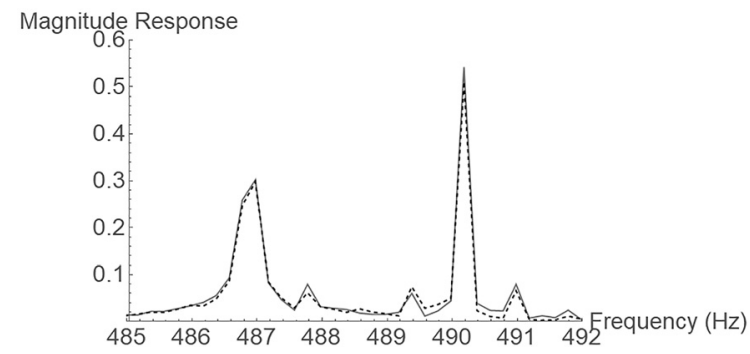

Fig. 7. Fourier spectrum corresponding to the Fig. 5 data.

in pressure by the finger on the glass rim due to difficulty in keeping the finger perfectly stationary.

\section{Struck rotating glass}

Our third scenario used a glass that was struck with a pen as it rotated on the turntable, creating a decaying signal.

We can observe from the Fourier spectrum in Fig. 11 that the two largest frequency peaks are $4.0 \mathrm{~Hz}$ apart, indicating that the pulsation frequency in Fig. 10 is $4.0 \mathrm{~Hz}$. Unlike the frequency factor of four times the glass's rotational frequency determined from Figs. 5 and 7, we find that the pulsation frequency in the struck glass case is only 2.24 times the glass's rotational frequency of $11.24 \mathrm{rad} / \mathrm{s}$ or $1.79 \mathrm{~Hz}$.

\section{DISCUSSION}

\section{A. Interference and standing waves}

When sinusoidal signals of two different frequencies are added together, the resultant beat frequency is exactly equal to the difference in frequency between the two signals. In the case of the rotating finger, we observe the converse: that a signal demonstrating a pulsating pattern of variations in intensity will have a Fourier spectrum containing two frequencies separated by the frequency of the original signal, as observed in Figs. 7 and 11. Rather than adding two different frequency signals together to obtain beats, the sound intensity fluctuations are instead created through interference. As the signals from the rotating wineglass antinodes interfere, they create a pulsating signal from which the two frequencies can be extracted.

The physical explanation for the results found in the case of the rotating finger arises from the rotation of the nodes and antinodes around the glass rim with respect to the two microphones. The four sources are thought to produce sound waves in the direction of vibrational motion of the wine glass rim. At the four antinode points, the wine glass rim moves in

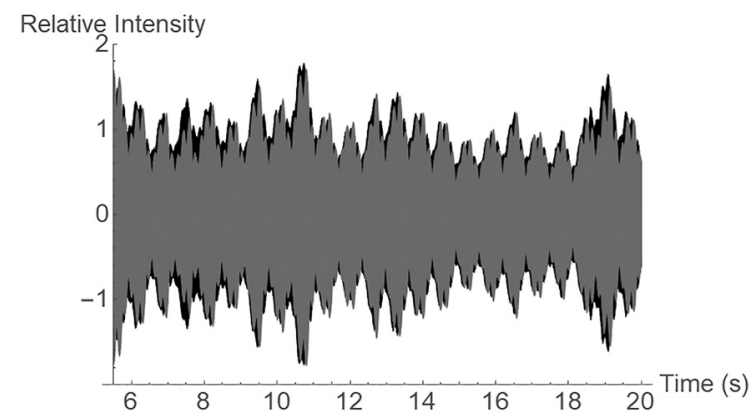

Fig. $8.45^{\circ}$ microphone offset: rotating glass at $9.80 \mathrm{rad} / \mathrm{s}$. 


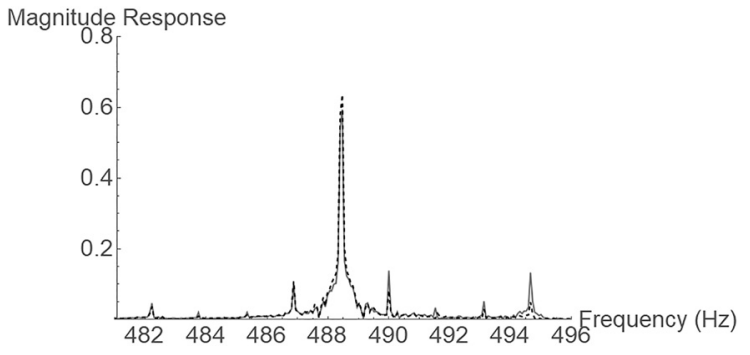

Fig. 9. Fourier spectrum of the Fig. 8 data showing a peak at the fundamental frequency of $488.8 \mathrm{~Hz}$, accompanied by symmetric side peaks.

the radial direction only. Therefore, with a moving finger in Fig. 12, the plane waves produced by the black antinode pair are orthogonal to the orientation of the microphone and therefore do not contribute to the total signal.

The gray antinode pair in Fig. 12, on the other hand, interferes to an extent determined by the wavelength of sound produced, as well as the $L_{1}$ and $L_{2}$ path lengths to create a net signal at the microphone.

When the four antinodes are rotated by an angle of $\pi / 4$, as in Fig. 13, the pair of sources corresponding to the equivalent $L_{1}$ and $L_{2}$ distances destructively interfere completely at the microphone due to the $\pi$ offset between the waves produced by the gray and black sources. Likewise, the two sources corresponding to the equivalent $L_{3}$ and $L_{4}$ distances also destructively interfere, resulting in a net zero signal amplitude at the microphone in the Fig. 13 orientation.

Therefore, as the four antinodes of the first harmonic rotate around the glass rim, the signal amplitude picked up by the microphone cycles between some positive amplitude $A$ and zero as a function of time. Differences in phase between the two microphones, then, vary as a function of their azimuthal angular displacement around the wine glass, with an angle of $45^{\circ}$ corresponding to a sound intensity phase shift of $\pi$.

On the short time scales illustrated in Fig. 4, phase shifts of 0 or $\pi$ in the waveforms result from the fact that the two antinode pairs produce signals that are out of phase. Amplitude zeroes occur in the Fig. 13 orientation, whereas amplitude maxima occur in the Fig. 12 orientation, with signs determined by which antinode pair is alligned with the receiver. Therefore, an arc traced between consecutive positive and negative maxima must go through a negative amplitude area, a zero point, and a positive amplitude area. If two receivers positioned $45^{\circ}$ apart reside in oppositely signed amplitude areas, the signs of the signals picked up by the two are opposite to each other (or shifted by $90^{\circ}$ ). Conversely, if both microphones reside in amplitude areas of the same sign, the two signals are in phase. Transitions between these two cases

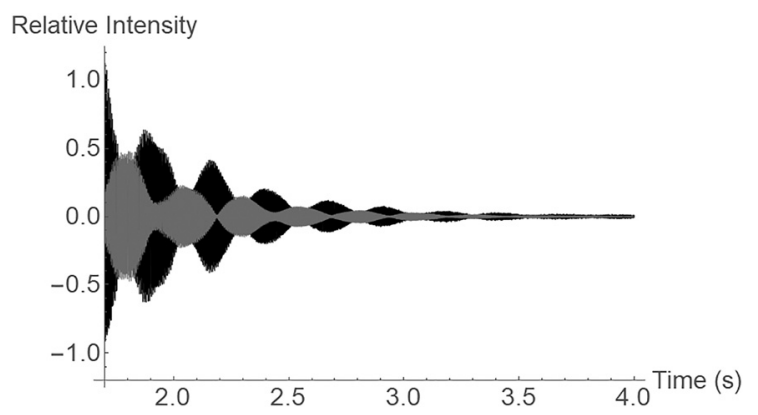

Fig. $10.45^{\circ}$ microphone offset: struck rotating glass at $11.24 \mathrm{rad} / \mathrm{s}$.

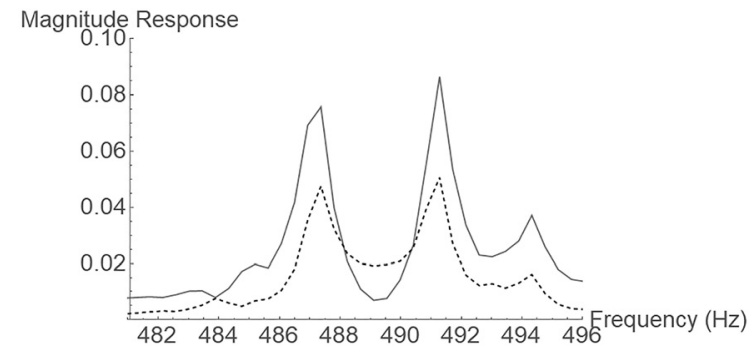

Fig. 11. Fourier spectrum of the signal in Fig. 10.

occur only when one of the receivers passes a zero-amplitude point, changing its signal amplitude's sign.

\section{B. Rotating rubber finger}

Students can develop and code models of different complexities to simulate these experiments. Here, we will show that even a simple model that can be easily implemented by students with minimal programming experience can be of great use to analyze a complicated system. To construct a model of the singing wine glass in Mathematica, we approximate antinodes as point sources, neglect all resonant frequencies except the fundamental, and assume that the amplitude A of sound emitted from each source remained constant. The largest sound intensity is emitted at the antinodes, and our simplified model ignores the other vibrating sections of the glass and approximates the system as the sum of the sound emitted by these four antinodes. We also treat the rim as the only part of the glass that oscillates, with sound waves being emitted from only the four antinode points on the rim. We do not include directivity in this simple model and assume that sound is emitted from each of the four antinode sources isotropically.

As illustrated in Fig. 14, the model uses four point sources spaced $90^{\circ}$ apart on the circumference of a glass rim of radius $\mathrm{R}$. We also neglect the inverse square decay of sound over distance in this simple model. The sources emit sound waves at the measured primary resonant frequency of the glass and rotate around the glass rim with the angular speed $\omega$ (in $\mathrm{rad} / \mathrm{s}$ ) of the rotating finger.

The horizontal and vertical distances between the microphone and each of the four point sources on the rim are defined in terms of $R$ and $r$, where $r$ is the distance between the center of the glass and the microphone. The sources are indexed $m=1$ through 4 in counterclockwise order (see Fig. 13). We assume the microphone points toward the center of the glass, along the $x$-axis in the plane of the rim. The $x$ and $y$ distances between source $m$ and microphone 1 vary sinusoidally with

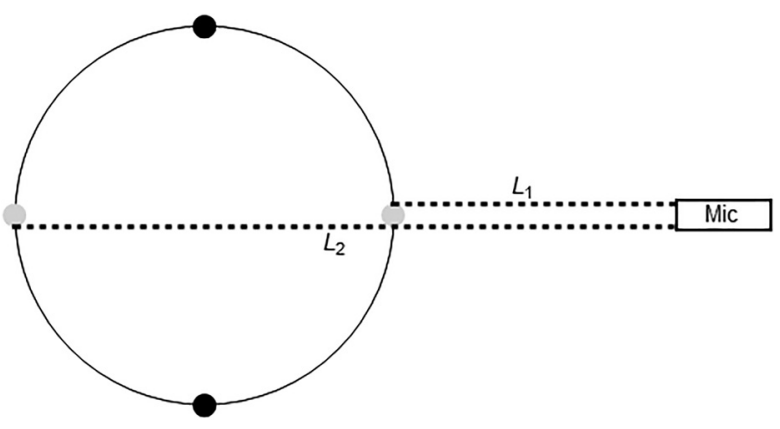

Fig. 12. Gray and black antinode pairs with no angular offset. 


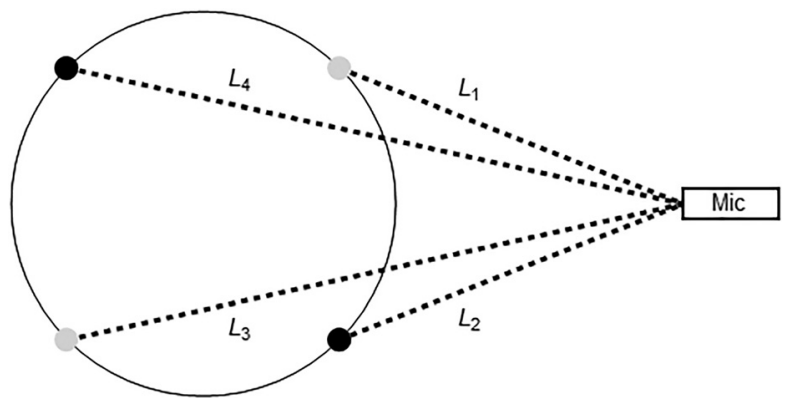

Fig. 13. Gray and black antinode pairs offset by an angle of $\pi / 4$.

time, $t$, based on the rotational speed, $\omega$, of the sources along the rim,

$$
\begin{aligned}
& x_{m}(t)=r-R \cos \left(\omega t+\frac{(m+1) \pi}{2}\right), \\
& y_{m}(t)=R \sin \left(\omega t+\frac{(m-1) \pi}{2}\right) .
\end{aligned}
$$

We find the four distances $d_{m}$ from their corresponding $x$ and $y$ displacements.

The contributions of the sound signals from each of the four antinodes can be calculated, where $s_{m}$ is the signal intensity due to the $m$ th antinode source of amplitude $\mathrm{A}$, the wavelength is $\lambda$ at the fundamental frequency $f$, and $S$ is the total summed signal intensity,

$$
\begin{aligned}
& s_{m}(t)=A \cos \left(\frac{2 \pi d_{m}}{\lambda}+m \pi+2 \pi f t\right), \\
& S(t)=\sum_{m=1}^{4} s_{m}(t) .
\end{aligned}
$$

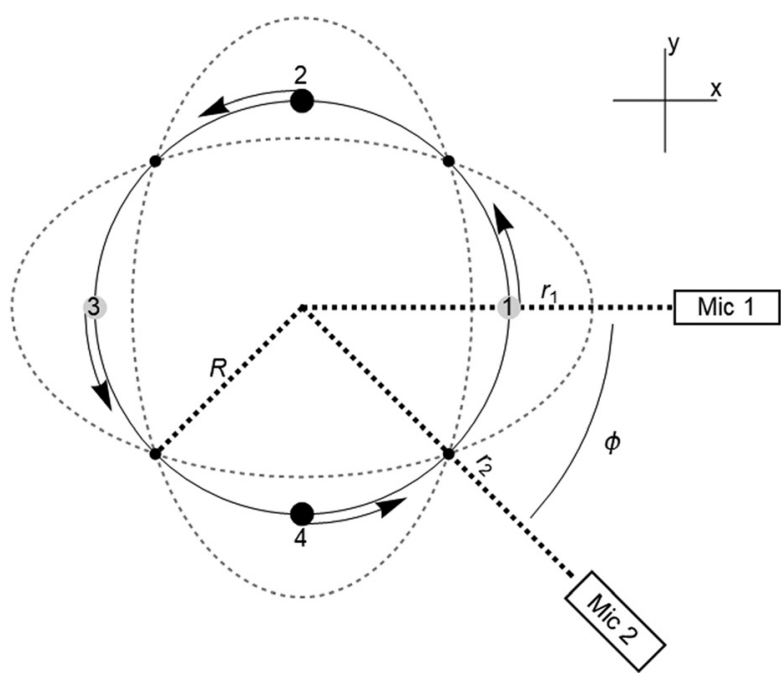

Fig. 14. A diagram of the experimental setup with important quantities labeled. $\mathrm{R}$ is the radius of the wine glass, $r_{1}$ and $r_{2}$ are the radial coordinates of the two microphones, and $\varphi$ is the azimuthal displacement between the two microphones. The large numbered gray and black dots correspond to the two out-of-phase antinode pairs, and the small black dots correspond to stationary nodes. Arrows illustrate the rotation of the nodes and antinodes around the glass, and dotted ellipses illustrate the exaggerated bending of the glass.

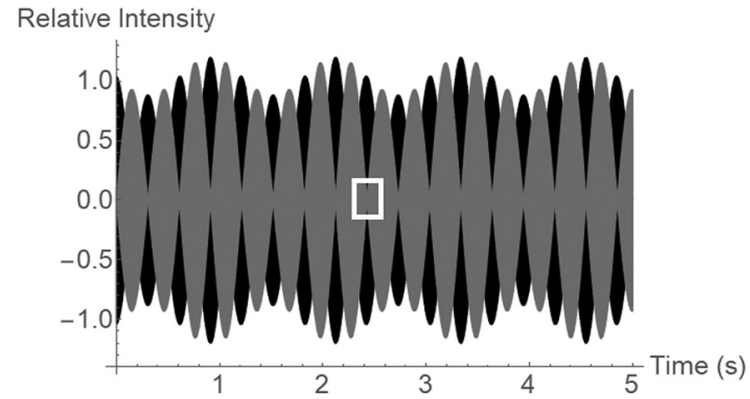

Fig. 15. An example model waveform using the values $\mathrm{R}=4.0 \mathrm{~cm}, \mathrm{r}=8.0 \mathrm{~cm}$, $\mathrm{f}=488.8 \mathrm{~Hz}, \alpha=0.15$, and $\omega=5.18 \mathrm{rad} / \mathrm{s}$. The parameters were chosen to closely model to the experimental data in Fig. 5. The boxed region is examined in more detail in Fig. 17.

We add a phase shift of $\pi$ to the signals of the $m=1$ and 3 sources to distinguish between the antinode pairs $m=\{1,3\}$ and $m=\{2,4\}$.

To model the waveform plot, we add the four signal contributions together as a function of time [Eq. (4)] and generate a fast Fourier frequency spectrum of the total simulated signal.

To simulate the secondary amplitude variation that is most visible in the Fig. 5 data, we multiply the total signal $S(t)$ by a sinusoidal factor $\sigma(t)$ with the same frequency as the rotational speed of the finger and a secondary amplitude $\alpha$,

$$
\sigma(t)=1-\alpha \sin (\omega t)
$$

The experimental and model datasets in Figs. 5 and 15 agree very closely, with both exhibiting a pulsation frequency of $3.3 \mathrm{~Hz}$. Students can also investigate how changing the azimuthal location of the microphone causes a shift in the signal's phase. This model also simulates the presence of a secondary pulsating envelope in the experimental data which repeats every fourth pulsation. We assume this signal to be due to the slight pressure variation by the rubber finger caused by the imperfectly flat nature of the glass rim.

In Fig. 16, we calculate the frequency spectrum of the model data from Fig. 15. From Fig. 16, the spectrum reveals a difference of $3.30 \mathrm{~Hz}$ between the two peaks in the Fourier spectrum. The frequency of $3.30 \mathrm{~Hz}$ models with the measured pulsation frequencies from Figs. 5 and 7. Similar to the relationship between beats and constituent frequencies, the distance between major peaks will scale exactly with the measured pulsation frequency. Students can use this type of experimental setup to find that the pulsation frequency indeed scales with the rotational speed of the glass as predicted by the simple model.

In both the experimental and modeled Fourier spectra (see Figs. 7 and 16), there are two pairs of small side peaks

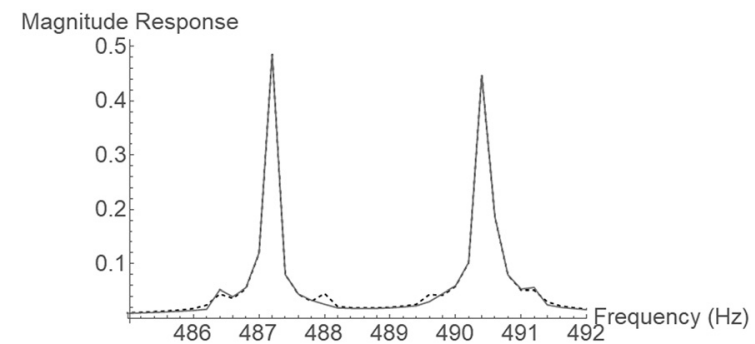

Fig. 16. The Fourier spectrum for one set of simulated data shown in Fig. 15. 


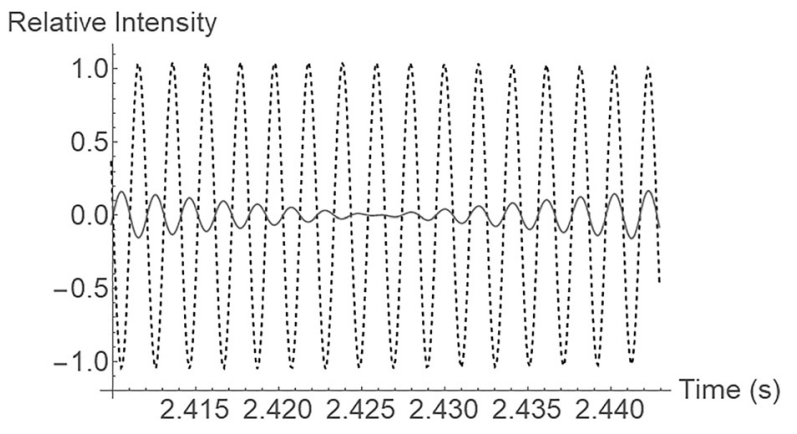

Fig. 17. Simulated data from Fig. 15 with waveforms from two microphones offset by $45^{\circ}$ centered on an amplitude zero of the solid-line signal.

located symmetrically on either side of the two major peaks with an average distance of $0.85 \mathrm{~Hz}$. This distance is approximately one quarter of the $3.3 \mathrm{~Hz}$ distance between the two major frequency peaks. This observation corresponds to the secondary sound amplitude variation which can be most easily seen in Fig. 5, in which sound intensity increases and decreases cyclically with a period corresponding to the rotational speed of the finger.

The model also accurately predicts phase shifts in the waveform. As shown in Fig. 4, the waveform phase shifts switch between 0 and $\pi$ every time each signal amplitude passes a minimum. This can also be seen in Fig. 17, in which a short time interval is examined near a simulated amplitude minimum occuring at $2.426 \mathrm{~s}$.

Upon passing the zero of the solid-line signal amplitude, the two waveforms abruptly switch from completely out of phase to in phase. Opposite transitions occur from in phase to out of phase behavior at minima of the dashed signal. While these phase transitions can occur over the span of several milliseconds in the experimental data, the idealized model data show these transitions distinctively without the experimental noise.

\section{Rotating glass with a stationary finger}

When the glass rotates rather than the finger, as in Fig. 8, a signal is produced that is not indicative of a beat-like sound, with a Fourier spectrum that includes only the fundamental resonance as its primary peak. This indicates that the nodes around the glass rim are stationary relative to the finger and microphones and do not rotate. The simple model would therefore result in a single Fourier peak at the fundamental frequency with an amplitude which is dependent on the angular distance of the microphone from the glass rim. Experimentally, slight periodic variation in amplitude creates symmetric peak pairs at integer multiple distances from the main peak, arising from periodic variation in the height of the glass rim, and therefore, finger pressure and amplitude. Other inconsistences in amplitude are caused by ambient noise and slight variations in the pressure on the rim arising from the difficulty of keeping one's finger pressure on the rim the same throughout. An additional setup or theoretical discussion about changing reference frames could explore the following case: A microphone rotating at the same rate as the wine glass with a stationary finger stimulating the wine glass to vibrate. With both, the wine glass and the microphone, being transferred in the same rotating reference frame, the pulsating sound pattern would reemerge.

\section{Struck rotating glass}

In the case of the struck rotating glass, a decaying pulsating sound pattern should be observed. According to G. H. Bryan, however, this case should produce nodes and antinodes that, instead of rotating with the glass, rotate at $3 / 5$ the angular speed of the body. ${ }^{9}$ This factor of $3 / 5$ is known as Bryan's factor, which is defined as the ratio of the angular speed of the vibrational pattern to the angular speed of the vibrating body - in this case, a wine glass. ${ }^{10}$ We observed in Figs. 10 and 11 that the data agree with Bryan's prediction. If the nodes and antinodes rotated with the body, four pulsations would be heard per revolution of the glass, which would result in a pulsation frequency of $7.16 \mathrm{~Hz}$. The frequency measured from Fig. 10 is $4.0 \mathrm{~Hz}, 55.9 \%$ of $7.16 \mathrm{~Hz}$, which is close to Bryan's factor of $3 / 5$.

While a factor of approximately $3 / 5$ can be expected for rotating wine glasses, Bryan's factor is not constant for all resonators and depends on a variety of factors, including the resonator's stiffness and geometry, as well as boundary conditions. ${ }^{11}$ Bryan's factor has been found to depend on the integrated tangential and radial displacements over the length of the resonator. ${ }^{12}$ Using this fact, Loveday and Rogers have determined that Bryan's factor of a resonating cyllinder can be decreased by restricting the vibration of either or both ends, thereby reducing total tangential motion. Additionally, it has been found that Bryan's factor can also be reduced by increasing the axial and rotational stiffness of the object, indicating that both composition and geometry play a significant role in the value of Bryan's factor for a particular resonator. ${ }^{12}$

Bryan's factor has an interesting application to aerospace engineering, in which such vibrations are used in gyroscopes. Linking the physics of a vibrating wine glass to modern technology used in space travel can be a surprising and exiting connection for students. Specifically, hemispherical resonator gyroscopes (or HRGs) detect rotation in the same way that we have observed-using the frequency of the acoustical varations of a flexing hemisphere to gauge rotational speed. ${ }^{13}$ Since the pulsations of the HRG are not alligned with its rotational speed, rotational speed can be determined by a sensor constrained to the same rotating reference frame as the resonator itself. Since their invention, HRGs have become the rotation sensors of choice for many aerospace applications due to their high accuracy and reliability, being first utilized in the NEAR program, as well as the NASA Cassini mission to Saturn launched in 1997.

\section{CONCLUSION}

In conclusion, we have observed that a finger rotating on the rim of a wine glass creates a beat-like tone, whereas a stationary finger on a rotating glass does not. We have shown through theory and modeling that the pulsating sound patterns in the tone created by a finger on the rim of a wine glass can be approximated by the interference between the four antinode sources rotating around the glass rim in two $\pi / 2$ offset pairs. This interference pattern rotates at the speed of the finger, causing regions of maximum and minimum amplitude to rotate past each microphone periodically, creating a pulsating sound intensity pattern. We have also shown that the difference between the rotational speed of the glass and the standing wave pattern in the case of a tapped rotating glass can be easily reproduced in an undergraduate lab. 
The phenomenon of rotating nodes and antinodes is rich in physics concepts that provide ample opportunities for lab projects for undergraduate physics or engineering students learning about acoustics, wave interference, Fourier spectra, and computational modeling.

\section{ACKNOWLEDGMENTS}

The authors want to thank Alex Chally for building the apparatus, Tetiana Korzun for the art work showing the experimental setup, Sara Chun, William Hahn, and Jay Jones for helping with the data collection, and Albert Bae, Erik Bodegom, and Robert Hauschild for their insights and advice.

\footnotetext{
${ }^{1}$ A. P. French, "In vino veritas: A study of wineglass acoustics," Am. J. Phys. 51, 688-694 (1983).

${ }^{2}$ J. W. S. Rayleigh, The Theory of Sound, 2nd ed. (MacMillan \& Company, London, 1894), Vol. I, pp. 383-389.

${ }^{3}$ R. T. Spurr, "The ringing of wine glasses," Wear 4, 150-153 (1961).
}

${ }^{4}$ H. Fenkner, "Ueber die Transversalschwingungen einerseits offener Metallcylinder," Ann. Phys. 244, 185-192 (1879).

${ }^{5}$ T. D. Rossing, "Chladni's law for vibrating plates," Am. J. Phys. 50, 271-274 (1982).

${ }^{6}$ T. D. Rossing, "Wine glasses, bell modes, and Lord Rayleigh," Phys. Teach. 28(9), 582-585 (1990).

${ }^{7}$ T. D. Rossing, "Acoustics of the glass harmonica," J. Acoust. Soc. Am. 95(2), 1106-1111 (1994).

${ }^{8}$ Logger pro, Vernier Software \& Technology, < https://www.vernier.com/ products/software/lp/>.

${ }^{9} \mathrm{G}$. H. Bryan, "On the beats in the vibrations of a revolving cylinder or bell," Cambridge Philos. Soc. 7(3), 101-111 (1890).

${ }^{10}$ S. V. Joubert, M. Y. Shatalov, and T. H. Fay, "Rotating structures and Bryan's effect,” Am. J. Phys. 77, 520-525 (2009).

${ }^{11}$ P. Loveday and C. Rogers, "Free vibration of elastically supported thin cylinders including gyroscopic effects," J. Sound Vib. 217(3), 547-562 (1998).

${ }^{12}$ M. Y. Shatalov and P. W. Loveday, "A theory of errors in vibratory gyroscopes," in Proceedings of the DGON Symposium on Gyro Technology, Stuttgart 1995, Chap. 15.

${ }^{13}$ D. M. Rozelle, "The hemispherical resonator gyro: From wineglass to the planets," Proceedings of the 19th AAS/AIAA Space Flight Mechanics Meeting, Savannah, Georgia, 2009, pp. 1157-1178.

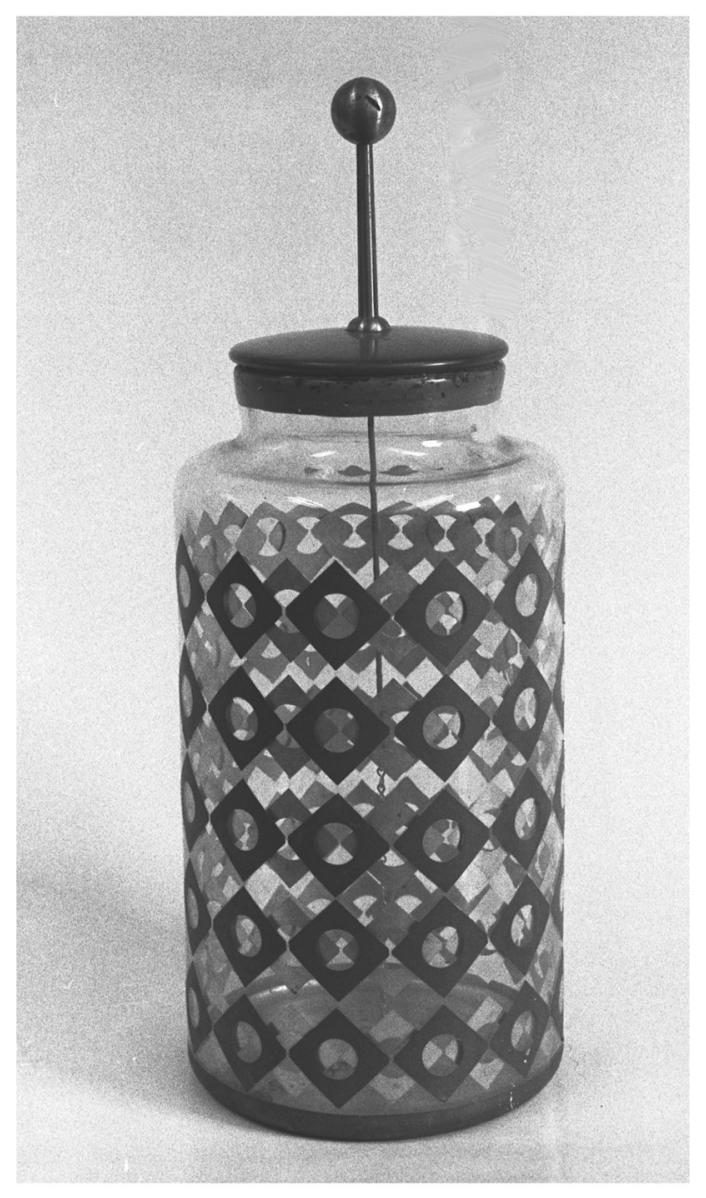

Diamond Jar

The diamond jar is a form of Leiden Jar, the original form of electrical condenser. The inner and outer coatings of the jar are made up of tinfoil diamonds with small spaces between their points. Holes punched in the coatings allow the sparks that jump between the points to be seen clearly as the jar is charged. The device is also called a Spangled Jar. This example is at Otterbein University. (Picture and text by Thomas B. Greenslade, Jr., Kenyon College) 